\title{
Talagrand Inequality on Free Path Space and Application to Stochastic Reaction Diffusion Equations*
}

\author{
Feng-Yu Wang ${ }^{a), c)}$, Tusheng Zhang ${ }^{b), d}$ \\ ${ }^{a)}$ Center for Applied Mathematics, Tianjin University, Tianjin 300072, China \\ ${ }^{b)}$ School of Mathematics, University of Manchester, Oxford Road, Manchester M13 9PL, U.K. \\ ${ }^{c}$ Department of Mathematics, Swansea University, Singleton Park, SA2 8PP, UK \\ d) School of Mathematics, University of Science and Technology of China, Hefei, China \\ wangfy@tju.edu.cn, tusheng.zhang@manchester.ac.uk
}

June 18, 2019

\begin{abstract}
By using a split argument due to [1], the transportation cost inequality is established on the free path space of Markov processes. The general result is applied to stochastic reaction diffusion equations with random initial values.
\end{abstract}

AMS subject Classification: 65G17, 65G60

Keywords: Stochastic reaction diffusion equations, Talagrand transportation cost inequality, free path space.

\section{Introduction}

Let $(E, \rho)$ be a metric space, and let $\mathscr{P}(E)$ be the class of all probability measures on $E$. The quadratic Warsserstein distance between $\mu_{1}, \mu_{2} \in \mathscr{P}(E)$ is defined by

$$
\mathbb{W}_{2}\left(\mu_{1}, \mu_{2}\right)=\inf _{\pi \in \mathscr{C}\left(\mu_{1}, \mu_{2}\right)}\left\{\int_{E \times E} \rho^{2}(x, y) \pi(\mathrm{d} x, \mathrm{~d} y)\right\}^{1 / 2}
$$

where $\mathscr{C}\left(\mu_{1}, \mu_{2}\right)$ is the space of all couplings of $\mu_{1}$ and $\mu_{2}$. In the study of Monge-Kontorovich optimal transportation problem, this distance is explained as the minimal cost to transport distribution $\mu_{1}$ into $\mu_{2}$ at the cost rate (cost function) $\rho$. Thus, an inequality involving $\mathbb{W}_{2}$

${ }^{*}$ Supported in part by NNSFC(11671372, 11431014, 11721101, 11771326, 11831014) 
is called a transportation cost inequality (TCI). Since the optimal transportation is usually unknown, in applications it is important to estimate $\mathbb{W}_{2}$ by easier to calculate quantities, for instance the relative entropy $H\left(\mu_{1} \mid \mu_{2}\right):=\int_{E}\left(\log \frac{\mathrm{d} \mu_{1}}{\mathrm{~d} \mu_{2}}\right) \mathrm{d} \mu_{1}$ if $\mu_{1}$ is absolutely continuous with respect to $\mu_{2}$, and $H\left(\mu_{1} \mid \mu_{2}\right):=\infty$ otherwise.

In 1996, Talagrand [18] established the following beautiful TCI for the standard Gaussian measure $\mu$ on $\mathbb{R}^{d}$ with $\rho(x, y)=|x-y|$ :

$$
\mathbb{W}_{2}(\nu, \mu)^{2} \leq 2 H(\nu \mid \mu), \quad \nu \in \mathscr{P}\left(\mathbb{R}^{d}\right),
$$

where the constant 2 is sharp. Since then, this type TCI has been intensively investigated and applied for various different distributions, and was linked to functional inequalities, concentration phenomena, optimal transport problem, and large deviations, see $[2,3,8,5,11$, 13, 19, 23] and references therein. Moreover, Talagrand type TCI has also been established on the path spaces of stochastic processes, see e.g. $[4,26,27]$ for diffusion processes on $\mathbb{R}^{d},[14]$ for multidimensional semi-martingales, $[1,20]$ for stochastic differential equations (SDEs) with memory, [5, 6, 22, 23, 24] for (reflecting) diffusion processes on Riemannian manifolds, [25] for SDEs driven by pure jump processes, and $[12,17]$ for SDEs with Lévy or fractional noises.

Recently, by using the Girsanov transformation argument developed from [4], the Talagrand inequality was established on the path space for solutions of stochastic reaction diffusion equations with deterministic initial values, see [10], [15]. In this paper, we aim to extend this result to the case with random initial values. In this case, the distribution of a solution is a probability measure on the free path space, where the initial value is not fixed. Since the Girsanov transformation does not change initial distributions, it does not work for probability measures with different initial distributions. However, two equivalent probability measures on the free path space may have different initial distributions. To overcome this difficulty, we will adopt a split argument used in [1] to reduce the problem to the case with deterministic initial value, to which the Girsanov transformation applies.

The remainder of the paper is organized as follows. In Section 2 we present a general result on the TCI for Markov processes with random initial values, which is then applied in Section 3 to stochastic reaction diffusion equations.

\section{A general result}

Let $(E, \rho)$ be a Polish space, and let $\left(P_{t}\right)_{t \geq 0}$ be the semigroup of a continuous Markov process on $E$. For any $T>0$ and $\mu \in \mathscr{P}(E)$, let $P^{\mu}$ denote the distribution of the Markov process up to time $T$ with initial distribution $\mu$; i.e. letting $P_{t}(x, \cdot)$ be the associated Markov transition kernel, $P^{\mu}$ is the unique probability measure on the free path space

$$
E_{T}:=C([0, T] ; E) \text { equipped with } \rho_{T}(\xi, \eta):=\sup _{t \in[0, T]} \rho\left(\xi_{t}, \eta_{t}\right),
$$

such that for any $0=t_{0}<t_{1} \cdots<t_{n}=T$ and $\left\{A_{i}\right\}_{0 \leq i \leq n} \subset \mathscr{B}(E)$,

$$
P^{\mu}\left(X_{t_{i}} \in A_{i}, 0 \leq i \leq n\right)=\int_{A_{0}} \mu\left(\mathrm{d} x_{0}\right) \int_{A_{1}} P_{t_{1}-t_{0}}\left(x_{0}, \mathrm{~d} x_{1}\right) \cdots \int_{A_{n}} P_{t_{n}-t_{n-1}}\left(x_{n-1}, \mathrm{~d} x_{n}\right),
$$


where $X_{t}, t \geq 0$ denotes the canonical coordinate process on the path space $E_{T}$. When $\mu=\delta_{x}$, the Dirac measure at $x \in E$, we simply denote $P^{\mu}=P^{x}$. Then

$$
P^{\mu}=\int_{E} P^{x} \mu(\mathrm{d} x), \quad \mu \in \mathscr{P}(E) .
$$

Let $\mathbb{W}_{2}$ and $\mathbb{W}_{2, T}$ be the Wasserstein distances induced by $\rho$ on $\mathscr{P}(E)$ and $\rho_{T}$ on $\mathscr{P}\left(E_{T}\right)$ respectively. We aim to establish the TCI for $P^{\mu}$ by using those for $\left\{P^{x}: x \in E\right\}$ and $\mu$.

T1 Theorem 2.1. Assume that for some constants $c_{1}, c_{2} \in(0, \infty)$ one has

$$
\begin{gathered}
\mathbb{W}_{2, T}\left(Q, P^{x}\right)^{2} \leq c_{1} H\left(Q \mid P^{x}\right), \quad x \in E, Q \in \mathscr{P}\left(E_{T}\right) \\
\mathbb{W}_{2, T}\left(P^{x}, P^{y}\right)^{2} \leq c_{2} \rho(x, y)^{2}, \quad x, y \in E
\end{gathered}
$$

If $\mu \in \mathscr{P}(E)$ satisfies

$$
\mathbb{W}_{2}(\nu, \mu)^{2} \leq c_{0} H(\nu \mid \mu), \quad \nu \in \mathscr{P}(E)
$$

for some constant $c_{0} \in(0, \infty)$, then

$$
\mathbb{W}_{2, T}\left(Q, P^{\mu}\right)^{2} \leq C H\left(Q \mid P^{\mu}\right), \quad Q \in \mathscr{P}\left(E_{T}\right)
$$

holds for $C=\left(\sqrt{c_{1}}+\sqrt{c_{0} c_{2}}\right)^{2}$. On the other hand, (2.5) implies (2.4) for $c_{0}=C$.

Proof. (1) We first deduce (2.5) from (2.4). Let $Q=F P^{\mu} \in \mathscr{P}\left(E_{T}\right)$ and $u_{0}: E_{T} \rightarrow E$ with $u_{0}(\xi)=\xi_{0}$. Then

$$
\left\{Q \circ u_{0}^{-1}\right\}(\mathrm{d} x)=p(x) \mu(\mathrm{d} x)=: \nu(\mathrm{d} x)
$$

holds for

$$
p(x):=\int_{E_{T}} F(\xi) P^{x}(\mathrm{~d} \xi), \quad x \in E .
$$

By the triangle inequality,

$$
\mathbb{W}_{2, T}\left(Q, P^{\mu}\right) \leq \mathbb{W}_{2, T}\left(Q, P^{\nu}\right)+\mathbb{W}_{2, T}\left(P^{\nu}, P^{\mu}\right) .
$$

Below we estimate these two terms respectively.

To estimate $\mathbb{W}_{2, T}\left(Q, P^{\nu}\right)$, we note that (2.1) implies

$$
\begin{aligned}
& \int_{E_{T}} f\left(\xi_{0}\right) F(\xi) P^{\mu}(\mathrm{d} \xi)=\int_{E} f(x) \mu(\mathrm{d} x) \int_{E_{T}} F(\xi) P^{x}(\mathrm{~d} \xi) \\
& =\int_{E} f(x) p(x) \mu(\mathrm{d} x)=\int_{E_{T}}(f p)\left(\xi_{0}\right) P^{\mu}(\mathrm{d} \xi), \quad f \in \mathscr{B}_{b}(E) .
\end{aligned}
$$


Therefore, letting $\mathbb{E}^{\mu}$ be the expectation with respect to $P^{\mu}$, we have

$$
p \circ u_{0}=\mathbb{E}^{\mu}\left(F \mid u_{0}\right) .
$$

Now, let

$$
F_{x}(\xi)=1_{\{p(x)>0\}} \frac{F(\xi)}{p(x)}, \quad x \in E, \xi \in E_{T} .
$$

By $(2.2)$, if $p(x)>0$ then

$$
\mathbb{W}_{2, T}\left(F_{x} P^{x}, P^{x}\right)^{2} \leq c_{1} P^{x}\left(F_{x} \log F_{x}\right) .
$$

So, for any $G, H \in \mathscr{C}$, where

$$
\mathscr{C}:=\left\{(G, H): G, H \in C_{b}\left(E_{T}\right), G(\xi) \leq H(\eta)+\rho_{T}(\xi, \eta)^{2} \text { for } \xi, \eta \in E_{T}\right\},
$$

we have

$$
\int_{E_{T}} F_{x} G \mathrm{~d} P^{x}-\int_{E_{T}} H \mathrm{~d} P^{x} \leq c_{1} \int_{E_{T}}\left(F_{x} \log F_{x}\right) \mathrm{d} P^{x}, \quad p(x)>0 .
$$

Integrating with respect to $\nu(\mathrm{d} x):=p(x) \mu(\mathrm{d} x)$ and using (2.1), we obtain

$$
\begin{aligned}
& Q(G)-P^{\nu}(H)=\int_{E_{T}} G \mathrm{~d} Q-\int_{E_{T}} H \mathrm{~d} P^{\nu} \\
& =\int_{E}\left\{\int_{E_{T}} F_{x} G \mathrm{~d} P^{x}-\int_{E_{T}} H \mathrm{~d} P^{x}\right\} p(x) \mu(\mathrm{d} x) \\
& \leq c_{1} \int_{E}\left\{\int_{E_{T}}\left(F_{x} \log F_{x}\right) \mathrm{d} P^{x}\right\} p(x) \mu(\mathrm{d} x) \\
& =c_{1} \int_{E_{T}}\left\{F \log F-F \log \mathbb{E}^{\mu}\left(F \mid u_{0}\right)\right\} \mathrm{d} P^{\mu} \\
& =c_{1} H\left(Q \mid P^{\mu}\right)-c_{1} \mathbb{E}^{\mu}\left[F \log \mathbb{E}^{\mu}\left(F \mid u_{0}\right)\right] \leq c_{1} H\left(Q \mid P^{\mu}\right),
\end{aligned}
$$

where the last step is due to the fact that

$$
\begin{aligned}
& \mathbb{E}^{\mu}\left[F \log \mathbb{E}^{\mu}\left(F \mid u_{0}\right)\right]=\mathbb{E}^{\mu}\left[\mathbb{E}^{\mu}\left(F \mid u_{0}\right) \log \mathbb{E}^{\mu}\left(F \mid u_{0}\right)\right] \\
& \geq \mathbb{E}^{\mu}\left[\mathbb{E}^{\mu}\left(F \mid u_{0}\right)\right] \log \mathbb{E}^{\mu}\left[\mathbb{E}^{\mu}\left(F \mid u_{0}\right)\right]=\mathbb{E}^{\mu}[F] \log \mathbb{E}^{\mu}[F]=0 .
\end{aligned}
$$

Therefore, by the Kontorovich dual formula, we arrive at

$$
\mathbb{W}_{2, T}\left(Q, P^{\nu}\right)^{2}=\sup _{(G, H) \in \mathscr{C}}\left\{Q(G)-P^{\nu}(H)\right\} \leq c_{1} H\left(Q \mid P^{\mu}\right) .
$$

On the other hand, by (2.3), for any $(G, H) \in \mathscr{C}$ we have

$$
\int_{E_{T}} G \mathrm{~d} P^{x}-\int_{E_{T}} H \mathrm{~d} P^{y} \leq c_{2} \rho(x, y)^{2}, \quad x, y \in E .
$$


Let $\pi \in \mathscr{C}(\nu, \mu)$ be the optimal coupling such that

$$
\mathbb{W}_{2}(\nu, \mu)^{2}=\int_{E \times E} \rho(x, y)^{2} \pi(\mathrm{d} x, \mathrm{~d} y)
$$

Integrating (2.10) with respect to $\pi(\mathrm{d} x, \mathrm{~d} y)$, and applying (2.1), we obtain

$$
\int_{E_{T}} G \mathrm{~d} P^{\nu}-\int_{E_{T}} H \mathrm{~d} P^{\mu}=\int_{E \times E}\left\{\int_{E_{T}} G \mathrm{~d} P^{x}-\int_{E_{T}} H \mathrm{~d} P^{y}\right\} \pi(\mathrm{d} x, \mathrm{~d} y) \leq c_{2} \mathbb{W}_{2}(\nu, \mu)^{2} .
$$

Combining this with the Kontorovich dual formula, and applying (2.4), we arrive at

$$
\mathbb{W}_{2, T}\left(P^{\nu}, P^{\mu}\right)^{2} \leq c_{2} \mathbb{W}_{2}(\nu, \mu)^{2} \leq c_{0} c_{2} \mu(p \log p)
$$

Since (2.1), (2.8) and Jensen's inequality imply

$$
\begin{aligned}
& \mu(p \log p)=\int_{E_{T}}\left\{\left(p \circ u_{0}\right) \log p \circ u_{0}\right\} \mathrm{d} P^{\mu} \\
& =\mathbb{E}^{\mu}\left[\mathbb{E}^{\mu}\left(F \mid u_{0}\right) \log \mathbb{E}^{\mu}\left(F \mid u_{0}\right)\right] \leq \mathbb{E}^{\mu}\left[\mathbb{E}^{\mu}\left(F \log F \mid u_{0}\right)\right]=H\left(Q \mid P^{\mu}\right),
\end{aligned}
$$

it follows from (2.11) that

$$
\mathbb{W}_{2, T}\left(P^{\nu}, P^{\mu}\right)^{2} \leq c_{0} c_{2} H\left(Q \mid P^{\mu}\right)
$$

Combining this with (2.7) and (2.9), we prove (2.5)

(2) To deduce (2.4) from $(2.5)$, for $\nu=p \mu$ we take $Q=\left(p \circ u_{0}\right) P^{\mu}$. Let $\Pi \in \mathscr{C}\left(Q, P^{\mu}\right)$ be the optimal coupling such that

$$
\mathbb{W}_{2, T}\left(Q, P^{\mu}\right)^{2}=\int_{E_{T} \times E_{T}} \rho_{T}^{2} \mathrm{~d} \Pi
$$

We have $\pi:=\Pi \circ\left(u_{0}, u_{0}\right)^{-1} \in \mathscr{C}(\nu, \mu)$, so that

$$
\begin{aligned}
& \mathbb{W}_{2}(\nu, \mu)^{2} \leq \int_{E \times E} \rho^{2} \mathrm{~d} \pi=\int_{E_{T} \times E_{T}} \rho^{2}\left(\xi_{0}, \eta_{0}\right) \Pi(\mathrm{d} \xi, \mathrm{d} \eta) \\
& \leq \int_{E_{T} \times E_{T}} \rho_{T}^{2}(\xi, \eta) \Pi(\mathrm{d} \xi, \mathrm{d} \eta)=\mathbb{W}_{2, T}\left(Q, P^{\mu}\right)^{2} .
\end{aligned}
$$

Combining this with (2.5) and noting that (2.1) implies

$$
H\left(Q \mid P^{\mu}\right)=\int_{E_{T}}\left\{\left(p \circ u_{0}\right) \log p \circ u_{0}\right\} \mathrm{d} P^{\mu}=\int_{E}(p \log p) \mathrm{d} \mu=H(\nu \mid \mu),
$$

we derive (2.4) for $c_{0}=C$. 


\section{TCI for stochastic reaction diffusion equations with random initial values}

Let $C_{0}([0,1])=\{u \in C([0,1]): u(0)=u(1)=0\}$. Consider the following SPDE on $C_{0}([0,1])$ :

$$
\left\{\begin{array}{l}
\mathrm{d} u_{t}(x)=\frac{1}{2} u_{t}^{\prime \prime}(x) d t+b\left(u_{t}(x)\right) d t+\sigma\left(u_{t}(x)\right) W(\mathrm{~d} t, \mathrm{~d} x), \quad x \in(0,1), \\
u_{t} \in C_{0}([0,1]), \quad t \geq 0,
\end{array}\right.
$$

where $W(\mathrm{~d} t, \mathrm{~d} x)$ is a space-time white noise on a complete probability space $(\Omega, \mathscr{F}, \mathbb{P})$ with natural filtration $\mathscr{F}_{t}$ generated by the Brownian sheet $\{W(t, x):(t, x) \in[0, \infty) \times[0,1]\}, u_{0}$ is a $C_{0}([0,1])$-valued random variable independent of $W$, and $b, \sigma: \mathbb{R} \rightarrow \mathbb{R}$ are locally bounded measurable functions. We say that an adapted, continuous process $\left\{u_{t}\right\}_{t \geq 0}$ on $C_{0}([0,1])$ is a solution to (3.1), if $\mathbb{P}$-a.s.

$$
\begin{aligned}
& \int_{0}^{1} u_{t}(x) \phi(x) \mathrm{d} x=\int_{0}^{1} u_{0}(x) \phi(x) \mathrm{d} x+\frac{1}{2} \int_{0}^{t} \mathrm{~d} s \int_{0}^{1} u_{s}(x) \phi^{\prime \prime}(x) \mathrm{d} x \\
& +\int_{0}^{t} \mathrm{~d} s \int_{0}^{1} b\left(u_{s}(x)\right) \phi(x) \mathrm{d} x+\int_{0}^{t} \int_{0}^{1} \sigma\left(u_{s}(x)\right) \phi(x) W(\mathrm{~d} s, \mathrm{~d} x), \quad t \geq 0, \phi \in C_{0}^{2}([0,1]),
\end{aligned}
$$

where $C_{0}^{2}([0,1]):=\left\{\phi \in C^{2}([0,1]): \phi(0)=\phi(1)=0\right\}$. According to [21], $u_{t}$ is a solution to (3.1) if and only if $\mathbb{P}$-a.s.

$$
u_{t}(x)=P_{t} u_{0}(x)+\int_{0}^{t} P_{t-s}\left\{b\left(u_{s}\right)\right\}(x) \mathrm{d} s+\int_{0}^{t} \int_{0}^{1} p_{t-s}(x, y) \sigma\left(u_{s}(y)\right) W(\mathrm{~d} s, \mathrm{~d} y), \quad t \geq 0,
$$

where $P_{t}$ and $p_{t}(x, y)$ are the Dirichlet heat semigroup and heat kernel generated by $\frac{1}{2} \Delta$ on $[0,1]$.

We will apply Theorem 2.1 to

$$
E:=C_{0}([0,1]), \quad E_{T}:=C([0, T] ; E)=C\left([0, T] ; C_{0}([0,1])\right),
$$

and $P^{\mu}$ being the distribution of the solution $\left(u_{t}\right)_{t \in[0, T]}$ with initial distribution $\mu \in \mathscr{P}(E)$. To this end, we need the following assumption.

(H) $\sigma$ is bounded, $b$ and $\sigma$ are Lipschitz continuous.

According to [21], when $b$ and $\sigma$ are Lipschitz continuous, (3.1) admits a unique solution for any (random) initial value $u_{0}$ on $E$. The boundedness of $\sigma$ was used in [15] to establish the TCI for solutions of (3.1) with deterministic initial values.

T2 Theorem 3.1. Assume $(\mathbf{H})$ and let $\mu \in \mathscr{P}(E)$. Then

$$
W_{2}\left(Q, P^{\mu}\right) \leq C H\left(Q \mid P^{\mu}\right), \quad Q \in \mathscr{P}\left(E_{T}\right)
$$

holds for some constant $C>0$ if and only if

$$
W_{2}(\nu, \mu) \leq c H(\nu \mid \mu), \quad \nu \in \mathscr{P}(E)
$$

holds for some constant $c>0$. 
Proof. In the present case, we have

$$
\begin{aligned}
& \rho(f, g)=\sup _{x \in[0,1]}|f(x)-g(x)|, \quad f, g \in E:=C_{0}([0,1]), \\
& \left.\rho_{T}(\xi, \eta)=\sup _{(t, x) \in[0, T] \times[0,1]}\left|\xi_{t}(x)-\eta_{t}(x)\right|, \quad \xi, \eta \in E_{T}:=C([0, T]] ; E\right) .
\end{aligned}
$$

According to [15], (2.2) holds for some constant $c_{1}>0$. So, by Theorem 2.1, it suffices to verify (2.3). Letting $u_{t}^{f}$ be the unique solution of (3.1) with $u_{0}=f \in E:=C_{0}([0,1])$, we only need to prove

$$
\mathbb{E}\left[\sup _{(t, x) \in[0, T] \times[0,1]}\left|u_{t}^{f}(x)-u_{t}^{g}(x)\right|^{2}\right] \leq c_{2} \sup _{x \in[0,1]}|f(x)-g(x)|^{2}, \quad f, g \in C_{0}([0,1])
$$

for some constant $c_{2}>0$. Indeed, since the law of $\left(u_{t}^{f}, u_{t}^{g}\right)_{t \in[0, T]}$ is a coupling of $P^{f}$ and $P^{g}$, we have

$$
\mathbb{W}_{2, T}\left(P^{f}, P^{g}\right)^{2} \leq \mathbb{E}\left[\rho_{T}\left(u^{f}, u^{g}\right)^{2}\right]=\mathbb{E}\left[\sup _{(t, x) \in[0, T] \times[0,1]}\left|u_{t}^{f}(x)-u_{t}^{g}(x)\right|^{2}\right] .
$$

Below we prove the estimate (3.6).

By (3.3) we have

$$
\mathbb{E}\left[\sup _{(t, x) \in[0, T] \times[0,1]}\left|u_{t}^{f}(x)-u_{t}^{g}(x)\right|^{2}\right] \leq 3 \rho(f, g)^{2}+3\left(I_{1}+I_{2}\right),
$$

where

$$
\begin{aligned}
& I_{1}:=\mathbb{E}\left[\sup _{(t, x) \in[0, T] \times[0,1]}\left|\int_{0}^{t} \int_{0}^{1} p_{t-s}(x, y)\left[b\left(u_{s}^{f}(y)\right)-b\left(u_{s}^{g}(y)\right)\right] \mathrm{d} s \mathrm{~d} y\right|^{2}\right], \\
& I_{2}:=\mathbb{E}\left[\sup _{(t, x) \in[0, T] \times[0,1]}\left|\int_{0}^{t} \int_{0}^{1} p_{t-s}(x, y)\left[\sigma\left(u_{s}^{f}(y)\right)-\sigma\left(u_{s}^{g}(y)\right)\right] W(\mathrm{~d} s, \mathrm{~d} y)\right|^{2}\right] .
\end{aligned}
$$

Noting that the Dirichlet heat kernel satisfies

$$
\sup _{x \in[0,1]} \int_{0}^{t} \mathrm{~d} s \int_{0}^{1} p_{t-s}(x, y)^{2} \mathrm{~d} y \leq \frac{\sqrt{2 t}}{\sqrt{\pi}}, \quad t>0,
$$

and due to $(\mathbf{H})$ we have

$$
|b(x)-b(y)| \vee|\sigma(x)-\sigma(y)| \leq K|x-y|, \quad x, y \in[0,1]
$$


for some constant $K>0$, by Hölder's inequality we obtain

$$
\begin{gathered}
I_{1} \leq K^{2} \mathbb{E}\left\{\operatorname { s u p } _ { ( t , x ) \in [ 0 , T ] \times [ 0 , 1 ] } \left[\left(\int_{0}^{t} \int_{0}^{1} p_{t-s}(x, y)^{2} \mathrm{~d} s \mathrm{~d} y\right)\right.\right. \\
\left.\left.\times\left(\int_{0}^{t} \int_{0}^{1}\left|u_{s}^{f}(y)-u_{s}^{g}(y)\right|^{2} d s d y\right)\right]\right\} \\
\leq \sqrt{\frac{2 T}{\pi}} K^{2} \int_{0}^{T} \mathbb{E}\left[\sup _{(r, y) \in[0, s] \times[0,1]}\left|u_{r}^{f}(y)-u_{r}^{g}(y)\right|^{2}\right] \mathrm{d} s .
\end{gathered}
$$

To estimate the term $I_{2}$, we recall the following inequality due to [15]: for any $T, \varepsilon>$ 0 , there exists a constant $C_{T, \varepsilon}>0$ such that for any adapted random field $\gamma(t, x)$ with $\mathbb{E}\left[\sup _{(s, x) \in[0, t] \times[0,1]}|\gamma(s, x)|^{2}\right]<\infty, t \geq 0$, we have

$$
\begin{aligned}
& \mathbb{E}\left[\sup _{(s, x) \in[0, t] \times[0,1]}\left|\int_{0}^{s} \int_{0}^{1} p_{s-r}(x, y) \gamma(r, y) W(\mathrm{~d} r, \mathrm{~d} y)\right|^{2}\right] \\
& \leq \varepsilon \mathbb{E}\left[\sup _{(s, x) \in[0, t] \times[0,1]}|\gamma(s, x)|^{2}\right]+C_{T, \varepsilon} \int_{0}^{t} \mathbb{E}\left[\sup _{(r, x) \in[0, s] \times[0,1]}|\gamma(r, x)|^{2}\right] \mathrm{d} r, \quad t \in[0, T] .
\end{aligned}
$$

Applying this to $\gamma(s, x)=\sigma\left(u_{s}^{f}(x)\right)-\sigma\left(u_{s}^{g}(x)\right)$ and using (3.8), we obtain that for any $\epsilon>0$,

$$
\begin{aligned}
I_{2} \leq & \epsilon \mathbb{E}\left[\sup _{(t, x) \in[0, T] \times[0,1]}\left|\sigma\left(u_{t}^{f}(x)\right)-\sigma\left(u_{t}^{g}(x)\right)\right|^{2}\right] \\
& +C_{T, \varepsilon} \mathbb{E} \int_{0}^{T} \sup _{y \in[0,1]}\left|\sigma\left(u_{s}^{f}(y)\right)-\sigma\left(u_{s}^{g}(y)\right)\right|^{2} \mathrm{~d} s \\
\leq & \epsilon K^{2} \mathbb{E}\left[\sup _{(t, x) \in[0, T] \times[0,1]}\left|u_{t}^{f}(x)-u_{t}^{g}(x)\right|^{2}\right] \\
& +C_{T, \varepsilon} K^{2} \int_{0}^{T} \mathbb{E}\left[\sup _{(r, y) \in[0, s] \times[0,1]}\left|u_{r}^{f}(y)-u_{r}^{g}(y)\right|^{2}\right] \mathrm{d} s, t \in[0, T] .
\end{aligned}
$$

So, setting

$$
Y(t):=\mathbb{E}\left[\sup _{(s, x) \in[0, t] \times[0,1]}\left|u_{s}^{f}(x)-u_{s}^{g}(x)\right|^{2}\right],
$$

which is finite for all $t \in[0, \infty)$ due to assumption (H), by combining (3.7)-(3.11) together we obtain

$$
Y(t) \leq 3 \rho(f, g)^{2}+3 \sqrt{\frac{2 T}{\pi}} K^{2} \int_{0}^{t} Y(s) \mathrm{d} s+3 \epsilon K^{2} Y(t)+3 C_{T, \epsilon} K^{2} \int_{0}^{t} Y(s) \mathrm{d} s, \quad t \in[0, T] .
$$

Choosing $\varepsilon=\frac{1}{6 K^{2}}$, we find a constant $c(T)>0$ such that

$$
Y(t) \leq 6 \rho(f, g)^{2}+c(T) \int_{0}^{t} Y(s) \mathrm{d} s, \quad t \in[0, T] .
$$


By Gronwall's inequality and $Y(t)<\infty$ for $t \geq 0$, this implies $(3.6)$ for $c_{2}=6 \mathrm{e}^{c(T) T}$.

To illustrate Theorem 3.1, we present examples of $\mu$ satisfying (3.5), such that (3.4) holds true. By [5, Theorem 3.1], the heat measure on the loop space $C_{0}([0,1])$ satisfies $(3.5)$. Next, by Gross [9], the log-Sobolev inequality holds for the Brownian bridge measure $\mu_{0}$ on $C_{0}([0,1])$ :

$$
\mu_{0}\left(F^{2} \log F^{2}\right) \leq 2 T \mu_{0}\left(\|D F\|_{H}^{2}\right)^{2}, \quad F \in \mathscr{D}(D), \mu_{0}\left(F^{2}\right)=1,
$$

where $(D, \mathscr{D}(D))$ is the Malliavin gradient operator and $\|h\|_{H}:=\left(\int_{0}^{T}\left|h_{t}^{\prime}\right|^{2} \mathrm{~d} t\right)^{\frac{1}{2}}$ is the CameronMartin norm. So, by a standard perturbation argument, the log-Sobolev inequality

$$
\mu\left(F^{2} \log F^{2}\right) \leq 2 T \mathrm{e}^{\mathrm{osc}(V)} \mu\left(\|D F\|_{H}^{2}\right)^{2}, \quad F \in \mathscr{D}(D), \mu\left(F^{2}\right)=1,
$$

holds for any probability measure $\mathrm{d} \mu=\mathrm{e}^{V} \mathrm{~d} \mu_{0}$ with $V \in \mathscr{B}_{b}\left(C_{0}([0,1])\right)$, where osc $(V):=$ $\sup V-\inf V$. According to [16, Theorem 1.10], this implies

$$
\tilde{\mathbb{W}}_{2}(\nu, \mu)^{2} \leq 2 T \mathrm{e}^{\mathrm{osc}(V)} H(\nu, \mu), \quad \nu \in \mathscr{P}\left(C_{0}([0,1])\right),
$$

where $\tilde{\mathbb{W}}_{2}$ is the Wasserstein distance induced by the Cameron-Martin distance on $E$. Since the Cameron-Martin distance is larger than the uniform distance $\rho,(3.5)$ holds for this class of measures $\mu$.

\section{References}

BWY [1] J. Bao, F.-Y. Wang, C. Yuan, Transportation cost inequalities for neutral functional stochastic equations, J. Anal. Appl. 32(2013), 457-475.

BGL [2] S. Bobkov, I. Gentil, M. Ledoux, Hypercontractivity of Hamilton-Jacobi equations, J. Math. Pure Appl. 80(2001), 669-696.

BG99 [3] S. Bobkov, F. Götze, Exponential integrability and transportation cost related to logarithmic Sobolev inequalities, J. Funct. Anal. 163(1999), 1-28.

DGW [4] H. Djellout, A. Guilin, L. Wu, Transportation cost-information inequalities for random dynamical systems and diffusions, Ann. Probab. 32(2004), 2702-2732.

FS [5] S. Fang, J. Shao, Transportation cost inequalities on path and loop groups, J. Funct. Anal. 218(2005), 293-317.

FW [6] S. Fang, F.-Y. Wang, Analysis on free Riemannian path spaces, Bull. Sci. Math. $\mathbf{1 2 9}(2005), 339-355$.

GL07 [7] N. Gozlan, C. Léonard, A large deviation approach to some transportation cost inequalities, Probab. Theory Related Fields 139(2007), 235-283. 
GRS11 [8] N. Gozlan, C. Roberto, P.-M. Samson, A new characterization of Talagrand's transportentropy inequalities and applications, Ann. Probab. 39(2011), 857-880.

Gross [9] L. Gross, Logarithmic Sobolev inequalities on loop groups, J. Funct. Anal. 102(1991), $268-313$.

KS [10] D. Khoshnevisan, A. Sarantsev, Talagrand concentration inequalities for stochastic partial differential equations, arXiv:1709.07098v3.

L [11] M. Ledoux, The Concentration of Measure Phenomenon, Mathematical Surveys and Monographs, American Mathematical Society, Providence, 2001.

M10 [12] Y. Ma, Transportation inequalities for stochastic differential equations with jumps, Stochastic Process. Appl. 120(2010), 2-21.

OV [13] F. Otto, C. Villani, Generalization of an inequality by Talagrand and links with the logarithmic Sobolev inequality, J. Funct. Anal. 173(2000), 361-400.

P11 [14] S. Pal, Concentration for multidimensional diffusions and their boundary local times, Probab. Theory Relat. Fields 154(2012), 225-254.

SZ [15] S. Shang, T. Zhang, Quadratic transportation cost inequality for stochastic reaction diffusion equations driven by multiplicative space-time white noise, arXiv:1904.13162.

Shao [16] J. Shao, Hamilton-Jacobi semi-groups in infinite-dimensional spaces, Bull. Sci. Math. 130(2006), 720-738.

S11 [17] B. Saussereau, Transportation inequalities for stochastic differential equations driven by a fractional Brownian motion, Bernoulli 18(2012), 1-23.

T96 [18] M. Talagrand, Transportation cost for Gaussian and other product measures, Geom. Funct. Anal. 6(1996), 587-600.

U [19] A.S. Üstünel, Introduction to Analysis on Wiener space, Lecture Notes in Math., Springer, 1995.

U10 [20] A.S. Üstünel, Transport cost inequalities for diffusions under uniform distance, in Stochastic Analysis and Related Topics pp. 203-214. Springer, 2012.

W [21] J. B. Walsh, An introduction to stochastic partial differential equations, Lecture Notes in Math. vol. 1180, 265-439, Springer, 1986.

W04 [22] F.-Y. Wang, Transportation cost inequalities on path spaces over Riemannian manifolds, Illinois J. Math 46(2002), 1197-1206.

W04b [23] F.-Y. Wang, Probability distance inequalities on Riemannian manifolds and path spaces, J. Funct. Anal. 206(2004), 167-190. 
W11 [24] F.-Y. Wang, Transportation-cost inequalities on path spaces over manifolds with boundary, Docum. Math. 18(2013), 297-322.

W10 [25] L. Wu, Transportation inequalities for stochastic differential equations of pure jumps, Ann. Inst. Henri Poincaré Probab. Stat. 46(2010), 465-479.

WZ04 [26] L. Wu, Z. Zhang, Talagrand's $T_{2}$-transportation inequality w.r.t. a uniform metric for diffusions, Acta Math. Appl. Sin. Engl. Ser. 20(2004), 357-364.

WZ06 [27] L. Wu, Z. Zhang, Talagrand's $T_{2}$-transportation inequality and log-Sobolev inequality for dissipative SPDEs and applications to reaction-diffusion equations, Chinese Ann. Math. Ser. B 27(2006), 243-262. 